\title{
On the conical refraction of hydromagnetic waves in plasma with anisotropic thermal pressure
}

\author{
David Tsiklauri * \\ Physics Department, University of Cape Town, Rondebosch 7r00, Republic of South Africa
}

(October 30, 2018)

\begin{abstract}
A phenomenon analogous to the conical refraction widely known in the crystalooptics and crystaloacoustics is discovered for the magnetohydrodynamical waves in the collisionless plasma with anisotropic thermal pressure. Angle of the conical refraction is calculated for the medium under study which is predicted to be $18^{\circ} 26^{\prime}$. Possible experimental corroborating of the discovered phenomenon is discussed.
\end{abstract}

52.35.Bj, 42.15.Gs

\section{INTRODUCTION}

A phenomenon of the conical refraction - a peculiar kind of light refraction in biaxial crystals taking place when a direction of a light beam coincides with some of the optical axes (binormals) of the crystal, was predicted theoretically by W.R. Hamilton even in 1832. He discovered this phenomenon basing on the Huygens-Fresnel principle (formulated 17 years earlier) applied for the biaxial crystals. In $1833 \mathrm{H}$. Lloyd proved experimentally existence of the phenomenon. Observation of the inner conical refraction in biaxial crystal is possible if the parallel, narrow beam of natural (nonpolarized) light falls along the optical axis (binormal) on a plate cut out from the crystal perpendicularly to the binormal. Propagating through the crystal the beam will form an empty cone with permanently changing linear polarization. Further, the phenomenon of the conical refraction was discovered for the elastic waves propagating along the acoustical axis of the crystal. For example, the inner conical refraction may take place when purely shearing waves propagate through the crystal along the third order symmetry axis (principal axis of symmetry [001] in triagonal crystals; direction [111] along the diagonal of a cube in the cubical crystals). In certain cases phenomenon of the acoustical conical refraction may be rather pronounced. The angle of the conical refraction (i.e., angle of deflection of the beam from the acoustical axis of the crystal) may have significant magnitude, for instance, in calcit $\mathrm{CaCO}_{3} \approx 30^{\circ}$, in quartz $\approx 17^{\circ}$ etc.

Resuming aforesaid, the phenomenon of the conical refraction in the crystals (optical and acoustical) is known for quite a long time and hence is completely studied. However, further display of the phenomenon is rather surprising: in Ref. [1] there was theoretically discovered the phenomenon analogous to the inner conical refraction for magnetosonic waves propagating in the isotropic plasma which is considered as a perfectly conducting fluid with adiabatical perturbations in it (see for brief description of the effect more accessible Ref. [2]). In particular [1], if the Alfven velocity coincides with the speed of sound then in the case of propagating of a quasiplane magnetosonic wave along the uniform magnetic field the beams corresponding to the wave will form an empty cone with the opening angle $53^{\circ} 08^{\prime}$, i. e. the angle of conical refraction for the magnetosonic waves in isotropic plasma was predicted to be $26^{\circ} 34^{\prime}$.

In the present paper, while considering adiabatical disturbances in collisionless plasma contained in a uniform external magnetic field and hence described by the theory of Chew, Goldberger and Low [3,4,5], is discovered phenomenon analogous to the inner conical refraction. Calculations showed that pending angle of the conical refraction for the magnetohydrodynamical (MHD) waves in the plasma with anisotropic thermal pressure should be $18^{\circ} 26^{\prime}$. The aim of this paper is to show theoretically that the phemomenon analogous to the inner conical refraction should also exist in anisotropic plasma medium and to encourage other authors to prove experimentally existence of the phenomenon. The beams are interpreted as lines along which the group velocity vector is directed.

In the concluding part of the paper there are discussed possibilities of experimental corroborating of the discovered phenomenon.

\footnotetext{
${ }^{*}$ On leave from the Department of Theoretical Astrophysics, Abastumani Astrophysical Observatory, Kazbegi str. N. $2^{a}$, Tbilisi 380060, Republic of Georgia
} 


\section{MAIN CONSIDERATION}

It is known $[4,5]$ that the linear MHD waves existing in the plasma described by the anisotropic thermal pressure obey to the following dispersion relation:

$$
\begin{gathered}
\omega^{2}=\frac{k^{2}}{2 \rho_{0}}\left[\frac{B_{0}^{2}}{4 \pi}+p_{\perp}\left(1+\sin ^{2} \theta\right)+2 p_{\|} \cos ^{2} \theta\right. \\
\pm \sqrt{\left.\left(\frac{B_{0}^{2}}{4 \pi}+p_{\perp}\left(1+\sin ^{2} \theta\right)-4 p_{\|} \cos ^{2} \theta\right)^{2}+4 p_{\perp}^{2} \sin ^{2} \theta \cos ^{2} \theta\right]}
\end{gathered}
$$

We further can rewrite eq. (1) in more convenient form introducing notations similar to those ones from Ref. [1]:

$$
a=\frac{B_{0}}{\sqrt{4 \pi \rho_{0}}}, \quad c_{\perp}=\sqrt{\frac{p_{\perp}}{\rho_{0}}}, c_{\|}=\sqrt{\frac{p_{\|}}{\rho_{0}}}
$$

where $a$ is the Alfven velocity, $c_{\perp}$ and $c_{\|}$are the sound speeds measured in perpendicular and parallel directions in respect to the uniform, external magnetic field $\vec{B}_{0}$. Doing so, we get:

$$
V^{2}=\frac{1}{2}\left[a^{2}+c_{\perp}^{2}\left(1+\sin ^{2} \theta\right)+2 c_{\|}^{2} \cos ^{2} \theta \pm \sqrt{\left(a^{2}+c_{\perp}^{2}\left(1+\sin ^{2} \theta\right)-4 c_{\|}^{2} \cos ^{2} \theta\right)^{2}+4 c_{\perp}^{4} \sin ^{2} \theta \cos ^{2} \theta}\right]
$$

where $V=\omega / k$ denotes phase velocity of the waves. Traditionally, here $\theta$ denotes an angle between $\vec{B}_{0}$ and wave vector $\vec{k}$.

Let us consider the special subcase of eq. (2) assuming that condition $c_{\perp}=\sqrt{2} c_{\|}$is fulfilled in the medium under study. It is worthwhile to note that the latter assumption leads to existence of so called "angular point" [1] on the surfaces formed by the phase velocities (both fast and slow MHD waves) just in the point of intersection of the ones with the direction of the magnetic field $\vec{B}_{0}$. Such a location of angular point, in turn, is responsible for the existence of the effect of conical refraction in the medim under study. The main guess was to find such a condition which would led to presence of the conical refraction in the anisotropic plasma medium. i.e. to discontinuous behavour of the vector of group velocity when $\theta$ tends to zero (see below). Since possessing such a condition we can rewrite eq.(2) as a following:

$$
V^{2}=\frac{1}{2}\left[a^{2}+2 c_{\perp}^{2} \pm \sqrt{\left(a^{2}+2 c_{\perp}^{2}\right)^{2}-4\left(\left[\frac{3}{2}\left(a^{2}+2 c_{\perp}^{2}\right)-c_{\perp}^{2}\right] c_{\perp}^{2} \cos ^{2} \theta-\frac{5}{4} c_{\perp}^{4} \cos ^{4} \theta\right)}\right]
$$

Basing on the latter expression we may calculate the group velocity of the waves. In general, the group velocity $\vec{u}$ has physical meaning of the wave packet propagation speed and can be found by simple formula $\vec{u}=\partial \omega / \partial \vec{k}$. The derivation of (3) results:

$$
\vec{u}=V \frac{\left[\left(1-\left[\left(5 c_{\perp}^{4}\right) /\left(4 V^{4}\right)\right] \cos ^{4} \theta\right)\right] \vec{N}-\left[\left(\cos \theta / V^{4}\right)\left(\left[\frac{3}{2}\left(a^{2}+2 c_{\perp}^{2}\right)-c_{\perp}^{2}\right] c_{\perp}^{2}-\frac{5}{2} c_{\perp}^{4} \cos ^{2} \theta\right)\right] \vec{A}}{1-\left[\left[\frac{3}{2}\left(a^{2}+2 c_{\perp}^{2}\right)-c_{\perp}^{2}\right] c_{\perp}^{2} \cos ^{2} \theta-\frac{5}{4} c_{\perp}^{4} \cos ^{4} \theta\right] / V^{4}}
$$

where we introduced unit vectors $\vec{N}=\vec{k} /|\vec{k}|$ and $\vec{A}=\vec{B}_{0} /\left|\vec{B}_{0}\right|$. To realize the physical picture geometrically it is worthwhile to state here following reasonings [1]: let multiply scalarly the latter formula by $\vec{N}$ :

$$
(\vec{u} \vec{N})=V
$$

i.e. projection of group velocity on the direction of wave nornal is equal to the phase velocity in the same direction. Multiplying eq. (5a) by $k$ one can get: $(\vec{u} \vec{k})=\omega$. Hence, for the infinitesimal changes of $\vec{u}, \vec{k}$ and $\omega$ the following relation is valid: $(\vec{u} \delta \vec{k})+(\vec{k} \delta \vec{u})=\delta \omega$. According to the definition of group velocity $(\vec{u} \delta \vec{k})=\delta \omega$. Therefore, $(\vec{k} \delta \vec{u})=0$, or in other terms

$$
(\vec{N} \delta \vec{u})=0
$$


The vector $\delta \vec{u}$ is located in the plane tangential to the surface (so called beam surface [1]) formed by the ends of vectors of the group velocities drawn away from the arbitrary point $O$. Therefore, eq. $(5 \mathrm{~b})$ implies that the plane, tangential to the beam surface, is perpendicular to the corresponding wave nornal. i.e. the beam surface is the envelope for the plane wave fronts, which were propagated from reference point $O$ in every directions per unit time. The latter statement in combination with eq.(5a) completely determines geometrical relationship between surface of phase velocities with the beam surface. However, it should be stressed that this geometrical relationship is valid only for the waves which phase velocities depend only on the direction and not on the length of the wave vector $\vec{k}$. It is enough to show that in such case eq. (5a) is valid. Let $V$ (phase velocity) be arbitrary function of wave vector $\vec{k}$. Then $\omega=k V(\vec{k})$ and hence, $\vec{u}=\partial \omega / \partial \vec{k}=V(\vec{k}) \vec{k} / k+k \partial V / \partial \vec{k}$. It is necessary and sufficient for condition (5a) to be hold that the $V$ must satisfy the following requirment: $\vec{k} \partial V / \partial \vec{k}=0$ or that is the same as $k_{i} \partial V / \partial k_{i}=0$. Therefore, one can conclude that $V$ must be homogeneous function of $k_{i}$ components of the zeroth order. The latter statement is equivalent to the requirement that function $V$ depends only on the direction of wave vector $\vec{k}$ and not on its length. As it can be seen clearly the dirpersion relations (1) and of course (2) and (3) satisfy this condition.

It is known [1] that since possessing surface formed by phase velocities one can construct geometrically the beam surface (formed by the ends of vectors of the group velocities) and vice versa. But instead of doing this it more convenient to carry out analitical calculation. Let us describe a direction of the group velocity $\vec{u}$ by an angle $\varphi$ between $\vec{u}$ and $\vec{B}_{0}$ (i.e., between $\vec{u}$ and $\vec{A}$ ). One can determine the angle $\varphi$ by the formula:

$$
\tan \varphi=\frac{\vec{u} \times \vec{A}}{\vec{u} \cdot \vec{A}}
$$

which with the aid of eq. (4) yields:

$$
\tan \varphi=\frac{\left[\left(1-\left[\left(5 c_{\perp}^{4}\right) /\left(4 V^{4}\right)\right] \cos ^{4} \theta\right)\right]}{1-\left[\left[\frac{3}{2}\left(a^{2}+2 c_{\perp}^{2}\right)-c_{\perp}^{2}\right] c_{\perp}^{2}-\frac{5}{2} c_{\perp}^{2} \cos ^{2} \theta+\frac{5}{4} c_{\perp}^{4} \cos ^{4} \theta\right] / V^{4}} \tan \theta
$$

By means of the eq. (6) we completely determined the direction of the group velocity $\vec{u}$ whereas its module can be calculated by expression:

$$
|\vec{u}|=V / \cos (\varphi-\theta)
$$

It is worthwhile to note that the eq. (7) can be obtained by using self-evident connection (5a) between the group velocity $\vec{u}$ and phase velocity $V$.

It is simple to show that expressions (4) and (6) have uncertain behavior as $0 / 0$ when $a=c_{\perp}$ (i.e., when $a=c_{\perp}=$ $\left.\sqrt{2} c_{\|}\right)$and $\theta$ tends to zero. Removing the uncertainty yields:

$$
\begin{gathered}
\tan \varphi(+0)=\lim _{\theta \rightarrow 0} \tan \varphi(\theta)= \pm \frac{1}{3} \\
u(+0)=\frac{\sqrt{10}}{3} c_{\perp}
\end{gathered}
$$

in eq. (8) sign "+" and "-" correspond to the slow and fast MHD waves respectively. The above treatment shows that when the wave normal $\vec{N}$ tends to reach a direction parallel to $\vec{B}_{0}$ (i. e. when $\theta$ tends to zero), vector of group velocity $\vec{u}$ does not takes the same direction, but its limiting position (when $\theta \rightarrow 0$ ) is the surface of an empty cone with opening angle $2 \varphi(+0)=2 \arctan (1 / 3)=36^{\circ} 52^{\prime}$. When $\theta$ is zero exactly then $\vec{u}$ is strictly parallel to $\vec{B}_{0}$. Such a discontinuous behavior of vector $\vec{u}$ can be explained by means of existence of so called [1] angular point which in the case of the anisotropic plasma exists when $a=c_{\perp}=\sqrt{2} c_{\|}$. (Note that for the waves in isotropic plasma the latter condition was rather simple [1] $a=c$ where $c$ denotes ordinary sound speed). So, when $a=c_{\perp}=\sqrt{2} c_{\|}$the angular point on the surfaces formed by the phase velocities (both fast and slow MHD waves) is located in the point of intersection of the ones with the direction of the magnetic field $\vec{B}_{0}$. Which, in turn leads to the discontinuous behaviur of $\vec{u}$ and causes the hole effect. In other terms the angular point is nothing else then point of intersection of the phase velocities of the fast and slow MHD waves. One can see from the Fig. 1 that when the condition $a=c_{\perp}$ is not satisfied than the polars of phase velocities (according to the dispersion relation (3)) do not have any points of intersection. It follows from the eq. (2) that when $a=c_{\perp}=\sqrt{2} c_{\|}$, then

$$
V^{2}=\frac{3 a^{2}}{2}\left[1 \pm \frac{\sin \theta}{3} \sqrt{4+5 \sin ^{2} \theta}\right] .
$$


From the eq.(10) we gather that when $\theta=0$ then $V_{+}=V_{-}$and hence the phase polars have two points of intersection which can be clearly seen from the Fig.2. It is apparent that in these points derivative of the phase velocities has uncertain behaviour. Removing of this uncertainity was done above. Result is clear now: in the anisotropic plasma for the linear MHD waves there should exist the effect analogous to the conical refraction and we worked out detailed explanation of the phenomenon.

\section{DISCUSSION}

Here in this section we discuss possibilities of experimental testing of existence of the phenomenon. Strictly speaking, the plane wave is idealization and real physical significance has quasiplane wave with small but finite angle of aperture. It should be emphasized that no real physical object corresponds to the wave with wave normal $\vec{N}$ exactly parallel to $\vec{B}_{0}(\theta=0)$, whereas in the case of propagation of quasiplane MHD wave with small but finite angle of aperture $(\theta \rightarrow 0)$ the narrow beam of such quasiplane waves, as it was shown above, will form an empty cone with opening angle $2 \varphi(+0)=36^{\circ} 52^{\prime}$ if $a=c_{\perp}=\sqrt{2} c_{\|}$condition is fulfilled. We may suppose the following experiment for demonstration of the phenomenon analogous to the conical refraction: Let us consider two collisionless plasma media contained in

a uniform external magnetic field $\vec{B}_{0}$. The media are separated by means of the plane perpendicular to the $\vec{B}_{0}$. Let choose external conditions in the second medium so that to satisfy the condition $a=c_{\perp}=\sqrt{2} c_{\|}$permanently. If we place a diaphragm between the first and second media then the beam of MHD waves propagating from the first medium towards the second one, just after passing the diaphragm will deflect from the direction of $\vec{B}_{0}$ and form an empty cone with angle of opening $2 \varphi(+0)=36^{\circ} 52^{\prime}$.

It is worthwhile to note that the effect of the conical refraction may be used for plasma diagnostics. It is clear that the due to the effect of the conical refraction intensity of wave which is registered by a detector placed on the path of propagation of the wave will show steep drop for certain value of the magnetic field which satisfies the condition

$$
\frac{B_{0}^{2}}{4 \pi}=p_{\perp}=2 p_{\|}
$$

Such a technique was proposed for isotropic plasma case in Ref.[6]

For the author is unknown whether the phenomenon analogous to the inner conical refraction of MHD waves in the anisotropic plasma was observed experimentally yet. However, one should take into account the difficulties in prepation of a plasma medium with permanently satified condition $a=c_{\perp}=\sqrt{2} c_{\|}$. The aim of this paper is to stimulate other authors, in spite of the techinacal difficulties, to prove the existence of the effect experimentally.

\section{ACKNOWLEDGEMENTS}

This research was supported, in part, by South African Foundation (FRD) for Research Development. It is my pleasure to express my gratitude to Prof. R.D. Viollier and Theoretical Physics Group of the Physics Department of the University of Cape Town for warm hospitality and creative atmosphere.

[1] D.V. Sivukhin, Magnitnaia gidrodinamica, 1, 35, 1966. (in Russian)

[2] A. I. Akhizer and Akhiezer, Plasma Electrodynamics, vol. 1: Linear Theory, (Pergamon Press, London, 1973)

[3] G. Chew, M. Goldberger, and F. Low, Proc. R. Soc. London ser. A 236, 112 (1956).

[4] N. A. Krall and A. W. Trivelpiece, Principles of Plasma Physics (McGraw-Hill, New-York, 1973).

[5] F. F. Cap, Handbook of Plasma Instabilities, vol. 1, (Academic Press, New York, 1976), p. 213

[6] R.V. Polovin and K.P. Cherkasova, High-frequensy Behaviour of Plasma (Naukova Dumka, Kiev, 1967) (in Russian) 


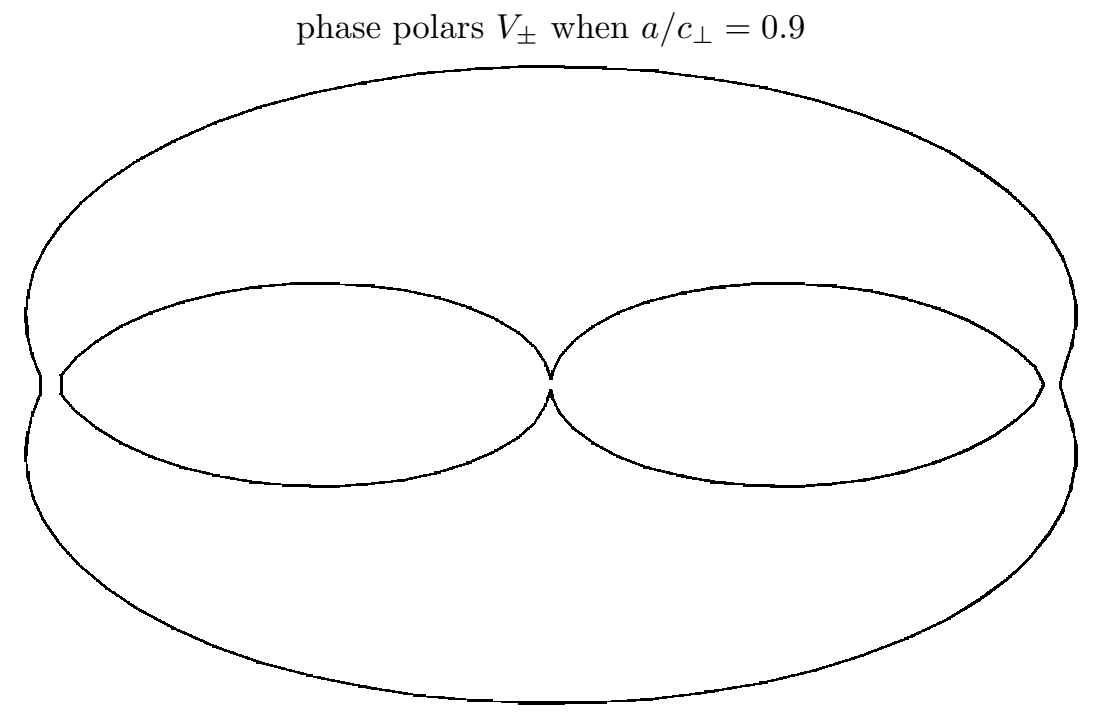

Fig. 1, graph of phase polars for slow and fast MHD waves according to eq. (3) for the case when $a / c_{\perp}=0.9$ i.e. near the degeneracy. 


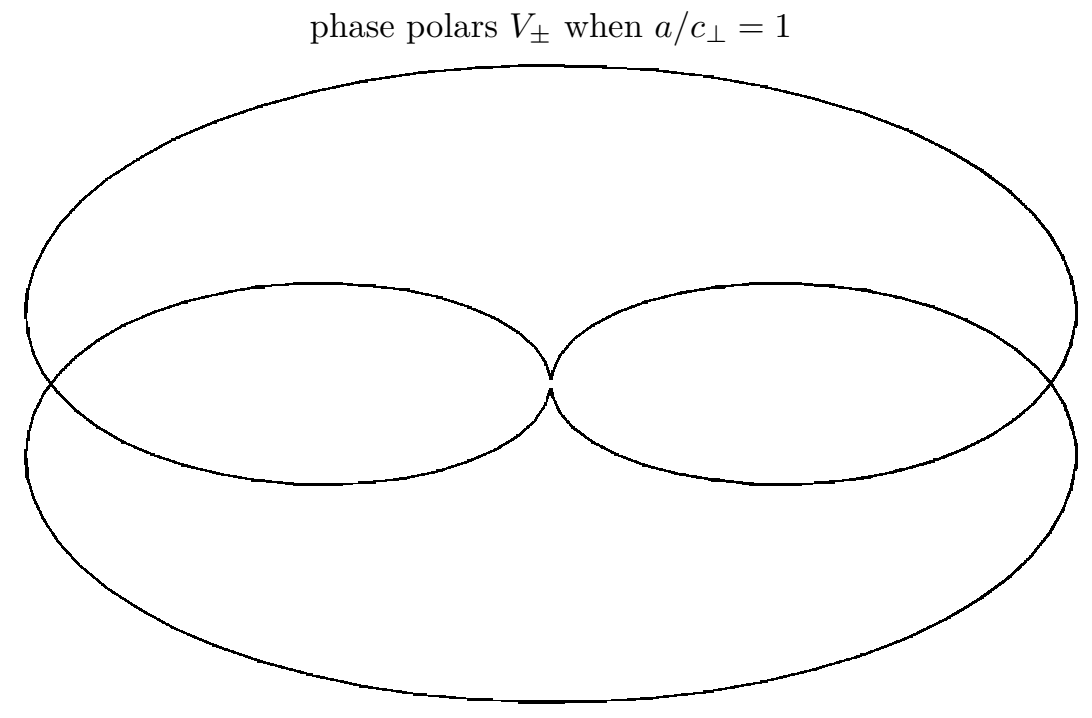

Fig. 2, graph of degenerate phase polars for slow and fast MHD waves according to eq. (3) or as well as eq.(10) for the case when $a / c_{\perp}=1$ 